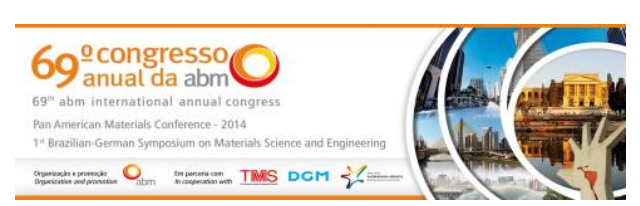

Tema: Metarlugia do pó

\title{
CARBETO DE TÂNTALO NANOMÉTRICO: OBTENÇÃO A BAIXA TEMPERATURA E CARACTERIZAÇÃO*
}

\author{
Maria José Santos Lima ${ }^{1}$ \\ Uilame Umbelino Gomes ${ }^{2}$ \\ Carlson Pereira de Souza ${ }^{2}$ \\ Maria Veronilda Macedo Souto ${ }^{1}$
}

\section{Resumo}

A pesquisa e o desenvolvimento de Materiais nanoestruturados vêm crescendo significativamente nos últimos anos. Estes materiais apresentam propriedades significativamente modificadas em comparação às dos materiais convencionais, devido às dimensões extremamente reduzidas dos cristalitos. $O$ carbeto de Tântalo $(\mathrm{TaC})$ é um material extremamente duro, apresentando elevada dureza, elevado ponto de fusão, elevada estabilidade química, boa resistência ao ataque químico e choque térmico e excelente resistência à oxidação e corrosão. Desta forma este trabalho teve como objetivo a obtenção de $\mathrm{TaC}$ nanoestruturado a partir do precursor tris(oxalato)oxitantalato de amônio hidratado, através de reação gás-sólido a baixa temperatura $\left(1.000^{\circ} \mathrm{C}\right)$ e curto tempo de reação. Os materiais obtidos foram caracterizados através de difração de Raios-X (DRX), Refinamento Rietveld, Microscopia Eletrônica de Varredura (MEV), Espectroscopia de Infravermelho (espectroscopia IV), Termogravimétrica (TG). Através das analises de DRX e do refinamento Reitiveld para o TaC com $S=1,1584$ observou-se a formação do carbeto de tântalo puro com estrutura cúbica e tamanho médio de cristalitos na ordem de 12,5 nanômetros.

Palavras-chave: Carbeto tântalo nanoestruturado; Precursores oxálicos; Reação gás sólido.

\section{NANOMETRIC TANTALUM CARBIDE: GETTING A LOW TEMPERATURE AND CARACTERIZAÇÃO}

\section{Abstract}

Electron Microscopy (SEM), infrared spectroscopy The research and development of nanostructured materials have been growing significantly in the last years. These materials have properties that were significantly modified as compared to conventional materials due to the extremely small dimensions of the crystallites. The tantalum carbide $(\mathrm{TaC})$ is an extremely hard material that has high hardness, high melting point, high chemical stability, good resistance to chemical attack and thermal shock and excellent resistance to oxidation and corrosion. Therefore, this study aimed to obtain $\mathrm{TaC}$ nanostructured from the precursor of tris (oxalate) hydrate ammonium oxitantalato, through gas-solid reaction at low temperature $\left(1,000^{\circ} \mathrm{C}\right)$ and short reaction time. The materials obtained were characterized by X-ray diffraction (XRD), Rietveld refinement, Scanning (IR), thermogravimetric (TG). Through the XRD analyses and the Reitiveld refinement of the $\mathrm{TaC}$ with $S=1.1584$, we observed the formation of pure tantalum carbide and cubic structure with average crystallite size on the order of 12.5 nanometers. Keywords: nanostructured tantalum carbide; oxalic precursors; gas-solid reaction.

1 Mestrando, Departamento de Ciências e Engenharia de Materiais, Universidade Federal do Rio Grande do Norte (UFRN), Natal, RN, Brasil.

2 Professor, Departamento de Ciências e Engenharia de Materiais, Universidade Federal do Rio Grande do Norte (UFRN), Natal, RN, Brasil.

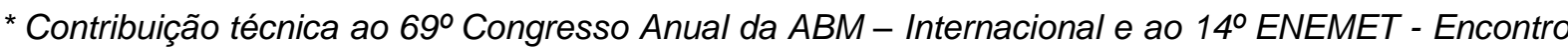
Nacional de Estudantes de Engenharia Metalúrgica, de Materiais e de Minas, 21 a 25 de julho de 2014, São Paulo, SP, Brasil.
} 


\section{INTRODUÇÃO}

Atualmente tem crescido significativamente os estudos para a produção e caracterização de materiais ultrafinos e nanoestruturados, o qual tem atraído à pesquisa fundamental e tecnológica nos últimos anos, devido às possibilidades de melhoria que as diversas propriedades que os materiais nanoestruturados podem ter em comparação aos materiais obtidos pelos processos convencionais. Esses novos métodos tem sido também aplicados a síntese de carbetos de metais refratários, os quais estão sendo desenvolvidos procurando produzir carbetos com propriedades adequadas para o uso na produção de carbetos cementados de alta qualidade e catalisadores mais seletivos.

Os carbetos são compostos binários de carbono os quais são classificados de acordo com o tipo de ligação química que ocorre na sua formação, podendo, assim, serem iônicos, covalentes ou metálicos. Esses carbetos possuem grande importância tecnológica, pois tem elevado ponto de fusão, boa resistência ao desgaste e extrema dureza. Devido a esses fatores, possuem grandes aplicabilidades como matrizes especiais nas indústrias aeroespacial, bélica, metalúrgica e química, e na constituição de carbetos cimentados, que são constituídos por carbetos de elevada dureza e possuem um metal ligante como aglomerante [1].

O carbeto de tântalo ( $\mathrm{TaC}$ ) é um material extremamente duro; é frequentemente adicionado ao compósito WC/Co para melhorar as propriedades físicas da estrutura sinterizada; atua como um inibidor de crescimento de grãos prevenindo a formação de grandes grãos e produzindo um material de excelente dureza; é usado como revestimento para moldes de aço na moldagem por injeção de ligas de alumínio; na produção de instrumentos cortantes com extrema resistência mecânica e dureza, é utilizado em brocas para ferramentas de corte. Tem a aparência de um pó marromcinza metálico com brilho e é altamente resistente à corrosão. Apresenta estrutura cristalina CFC, alta dureza, comparável com a do $\mathrm{NbC}$ e VC, e alto ponto de fusão (cerca de $3880^{\circ} \mathrm{C}$ ). Pode ser produzido ou pelo aquecimento de uma mistura de Ta e C (reação dependente de difusão no estado sólido) ou por aquecimento de uma mistura de $\mathrm{C}$ e $\mathrm{Ta}_{2} \mathrm{O}_{5}$ (redução de resíduos sólidos reagentes seguidos de carburação), sendo que este último é o método mais comum. O método tradicional de obtenção de carbetos é a partir dos seus óxidos metálicos, o que exige temperaturas elevadas e condições apropriadas que dificultam a obtenção desses carbetos com propriedades adequadas a catalise heterogênea.

Este trabalho teve como objetivo sintetizar o precursor tris(oxalato)oxitantalato de amônio hidratado e o carbeto de tântalo por meio de reação gás-sólido em reator tubular a baixa temperatura e tempo de reação e caracterizá-los por meio Difração de Raios-X (DRX), Refinamento Rietveld, Microscopia Eletrônica de Varredura (MEV), Analise Termogravimétrica (TG)) e Espectroscopia de Absorção na Região do Infravermelho (IV).

\section{MATERIAIS E MÉTODOS}

Com base na metodologia de Medeiros [2], o precursor tris(oxalato)oxitantalato de amônio hidratado foi produzido a partir de $4 \mathrm{~g}$ de óxido de tântalo e $40 \mathrm{~g}$ de bissulfato de potássio pesados numa balança analítica, e misturando $\mathrm{Nb}_{2} \mathrm{O}_{5}$ e $\mathrm{KHSO}_{4}$ numa razão de 1:10 através de almofariz e pistilo. Posteriormente, fez-se a fusão num cadinho de platina, resistente a altas temperaturas, com auxílio do bico

\footnotetext{
* Contribuição técnica ao $69^{\circ}$ Congresso Anual da ABM - Internacional e ao 14ํㅡㄹ ENEMET - Encontro Nacional de Estudantes de Engenharia Metalúrgica, de Materiais e de Minas, 21 a 25 de julho de 2014, São Paulo, SP, Brasil.
} 


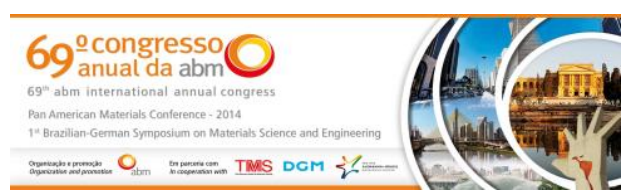

de Bunsen. Após a finalização da fusão foi obtido um líquido homogêneo de coloração rubra. O líquido foi transferido para um recipiente de porcelana a fim de ser resfriado e cristalizado. O material solidificado foi triturado com auxílio de almofariz e pistilo até a obtenção de um pó homogêneo de baixa granulometria.

Em seguida, adicionou-se aproximadamente $80 \mathrm{~mL}$ de ácido sulfúrico concentrado e quente. Foi adicionado aproximadamente $325 \mathrm{~mL}$ de hidróxido de amônio com auxílio de uma bureta até o pH atingir uma faixa entre 8 e 9. Posteriormente, houve lavagem com uma solução de ácido acético a $1 \%$ e em seguida, foi feita a complexação com uma solução a $65^{\circ} \mathrm{C}$ de ácido oxálico e oxalato de amônio com razão de $1: 3$ entre o tântalo e os íons oxalato, após a complexação a solução resultante ficou em repouso por 24 horas. A água da solução foi evaporada a uma temperatura de $80^{\circ} \mathrm{C}$, dando origem ao precursor, um sólido cristalino que foi triturado com auxílio de almofariz e pistilo até a obtenção de um pó homogêneo de baixa granulometria e seco em estufas por 24 horas.

O carbeto de tântalo foi produzido em baixa temperatura $\left(1000^{\circ} \mathrm{C}\right)$ a partir do precursor tris(oxalato)oxitantalato de amônio hidratado macerado em almofariz. $\mathrm{O}$ hidrogênio $\left(\mathrm{H}_{2}\right)$ e o metano $\left(\mathrm{CH}_{4}\right)$ foram utilizados como gases redutores e fonte de carbono respectivamente. As reações entre o precursor e a mistura de metano e hidrogênio foram efetuadas em um forno resistivo, composto de um reator de leito fixo de alumina. Para acomodar a amostra de precursor no reator de leito fixo foi utilizada uma barquinha de alumina, utilizando para todos os ensaios uma massa de 2 gramas, que foi introduzida no tubo de alumina até a parte central do forno. Após o fechamento do tubo, o mesmo foi lavado por alguns minutos com argônio a fim de eliminar todo o oxigênio presente. Em seguida, foram ajustados os fluxos dos gases reagentes (metano e hidrogênio) e a mistura gasosa foi circulada através do reator.

As reações de decomposição-redução-carbonetação foram feitas na temperatura de $1000^{\circ} \mathrm{C}$ e no tempo de isoterma de 120 minutos. Foi utilizada uma vazão de fluxo de $1 \mathrm{~L} / \mathrm{h}$ de metano refrente a $5 \%$ e $19 \mathrm{~L} / \mathrm{h}$ de hidrogênio, referente a $95 \%$ da mistura do fluxo gasoso [2]. Ao final da reação de decomposição-redução-carbonetação, o fluxo de gases reagentes foi trocado por um fluxo de argônio (10 L/h) e este fluxo foi mantido até a temperatura ambiente, quando as amostras foram retiradas.

\section{RESULTADOS E DISCUSSÃO}

\subsection{Caracterização do Precursor Oxálico de Tântalo}

O precursor oxálico de tântalo foi caracterizado por analise termogravimétrica (TG), espectroscopia de absorção na região do infravermelho (IV) e microscopia eletrônica de varredura (MEV).

\subsubsection{Espectroscopia de absorção na região do infravermelho do precursor}

A Figura 1 mostra o espectro de absorção na região do infravermelho do precursor oxálico de Ta. $\mathrm{O}$ espectro apresenta uma banda de absorção larga e de intensidade media na região de $3400 \mathrm{~cm}-1$ a $2950 \mathrm{~cm}-1$ em aproximadamente $3150 \mathrm{~cm}-1$ a qual pode ser atribuída ao modo de estiramento $\mathrm{v}(\mathrm{OH})$ da água de hidratação [3]. A banda apresentada na região de $1800 \mathrm{~cm}-1$ a $1550 \mathrm{~cm}-1$ em aproximadamente $1790 \mathrm{~cm}-1$ refere-se aos grupos oxalatos coordenados ao tântalo. Os modos de estiramento simétrico e assimétrico observados em 1790 - $2925 \mathrm{~cm}-1$ correspondem aos modos vibracionais do grupo oxalato. Nas regiões de $1235 \mathrm{~cm}-1$ e 1360 as bandas referem-se aos grupos iônicos $\mathrm{C}-\mathrm{O}$ e $\mathrm{C}=\mathrm{O}$ fato que acentua as

\footnotetext{
* Contribuição técnica ao $69^{\circ}$ Congresso Anual da ABM - Internacional e ao 14ํㅡㄹ ENEMET - Encontro Nacional de Estudantes de Engenharia Metalúrgica, de Materiais e de Minas, 21 a 25 de julho de 2014, São Paulo, SP, Brasil.
} 


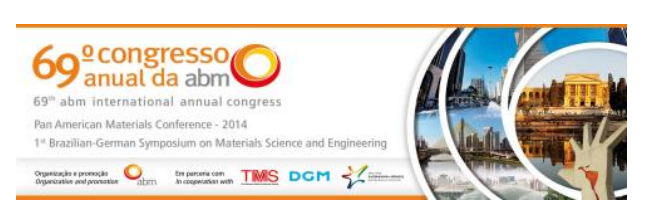

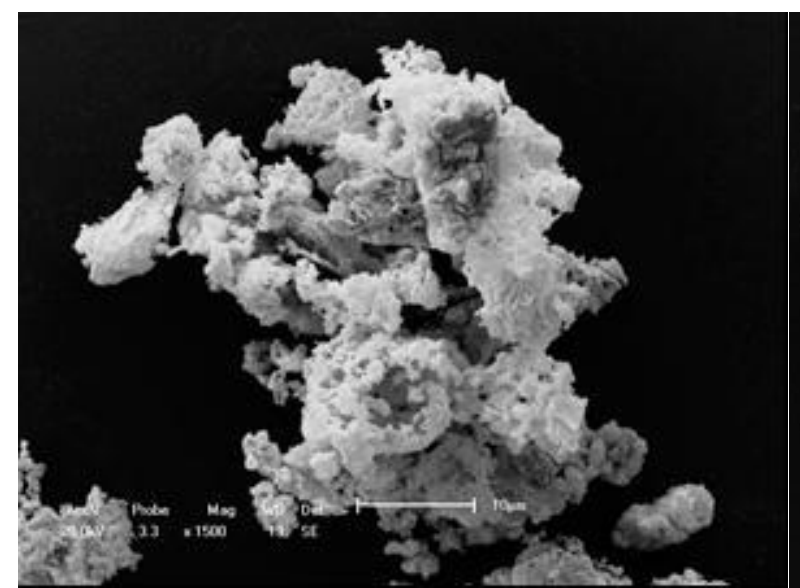

(a)

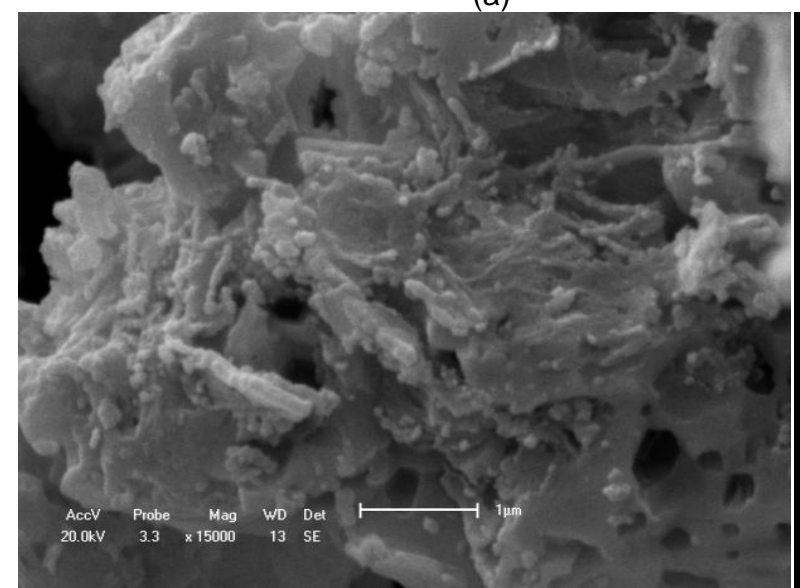

(c)

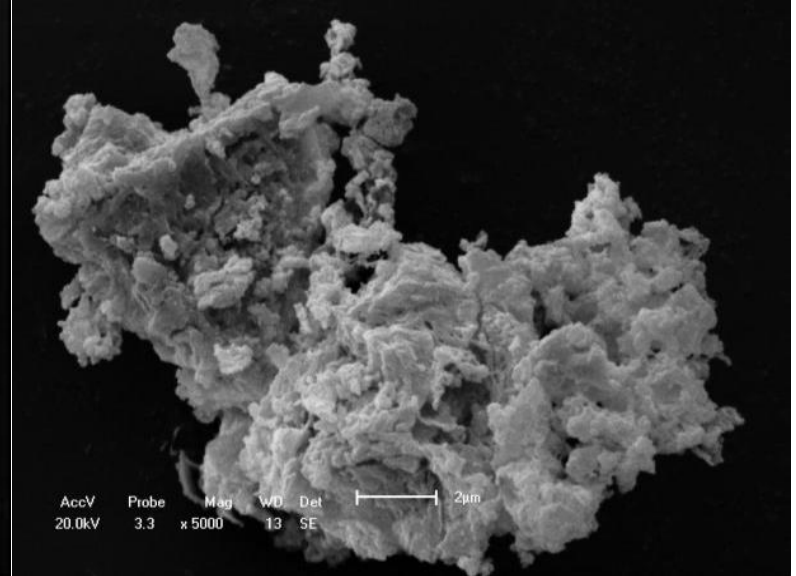

(b)

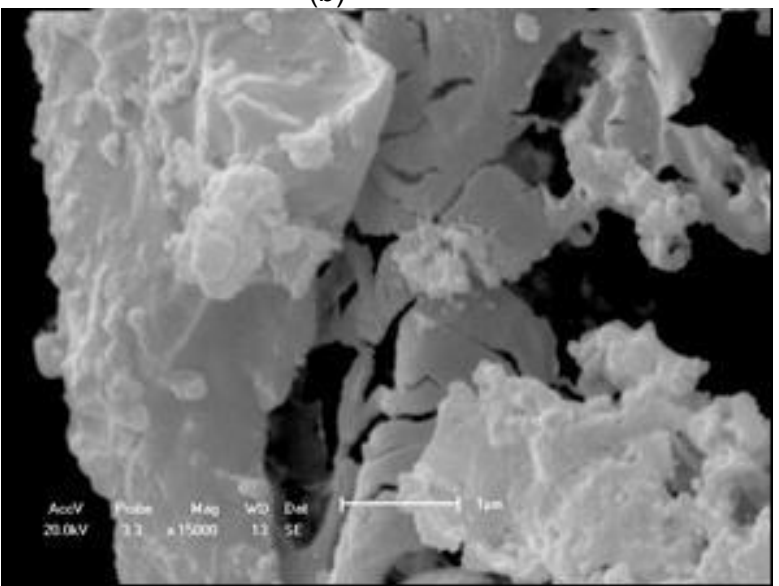

(d)

Figura 3. Microscopia Eletrônica de Varredura (MEV) do precursor oxálico de tântalo (1500x e 5000x e 15000x).

\subsection{Caracterização do Carbeto de Tântalo Nanométrico}

\subsubsection{DRX e método Rietveld do carbeto}

Os resultados obtidos por DRX após o processo de carbonetação indicaram a formação do carbeto de tântalo, apresentando intensidades nos ângulos de difração, mostrando que os $\mathrm{TaC}$ produzidos experimentalmente são puros. O refinamento Rietveld, indicou a presença do carbeto de tântalo de estrutura cúbica de face centrada (CFC), e permitiu determinar os parâmetros cristalográficos das amostras e o desvio em relação aos dados da literatura e o tamanho médio das partículas.

$\mathrm{Na}$ Figura 4, são apresentados os padrões experimentais de difração de Raios-X dos carbetos, identificando-se bem os picos característicos de TaC. Isso mostra que o processo de síntese via gás-sólido adotado nesse trabalho foi eficaz. Como pode ser visto, a síntese de TaC apresenta picos característicos e estrutura CFC. Além disso, observa-se picos bem largos. Fato que caracteriza a formação do carbeto de tântalo nanoestruturado. O refinamento para as amostras revelaram a formação do cristal na forma cubica e com os seguintes parâmetros cristalográficos: a $(\AA)=4,4495564$ b $(\AA)=4,4495564$, c $(\AA)=4,4495564$, Alpha $\left(^{\circ}\right)=90,0000$, Beta $\left(^{\circ}\right)=90,0000$, Gama $\left({ }^{\circ}\right)=90,0000$, Volume da célula $=4,4495564$, obteve um desvio igual a $S=1,1584$ e tamanho médio de cristalito igual $12,9 \mathrm{~nm}$. Na Tabela 3 são apresentados alguns dos principais dados referentes ao refinamentos realizado para o carbeto de tântalo.

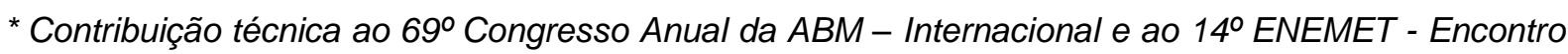
Nacional de Estudantes de Engenharia Metalúrgica, de Materiais e de Minas, 21 a 25 de julho de 2014, São Paulo, SP, Brasil.
} 


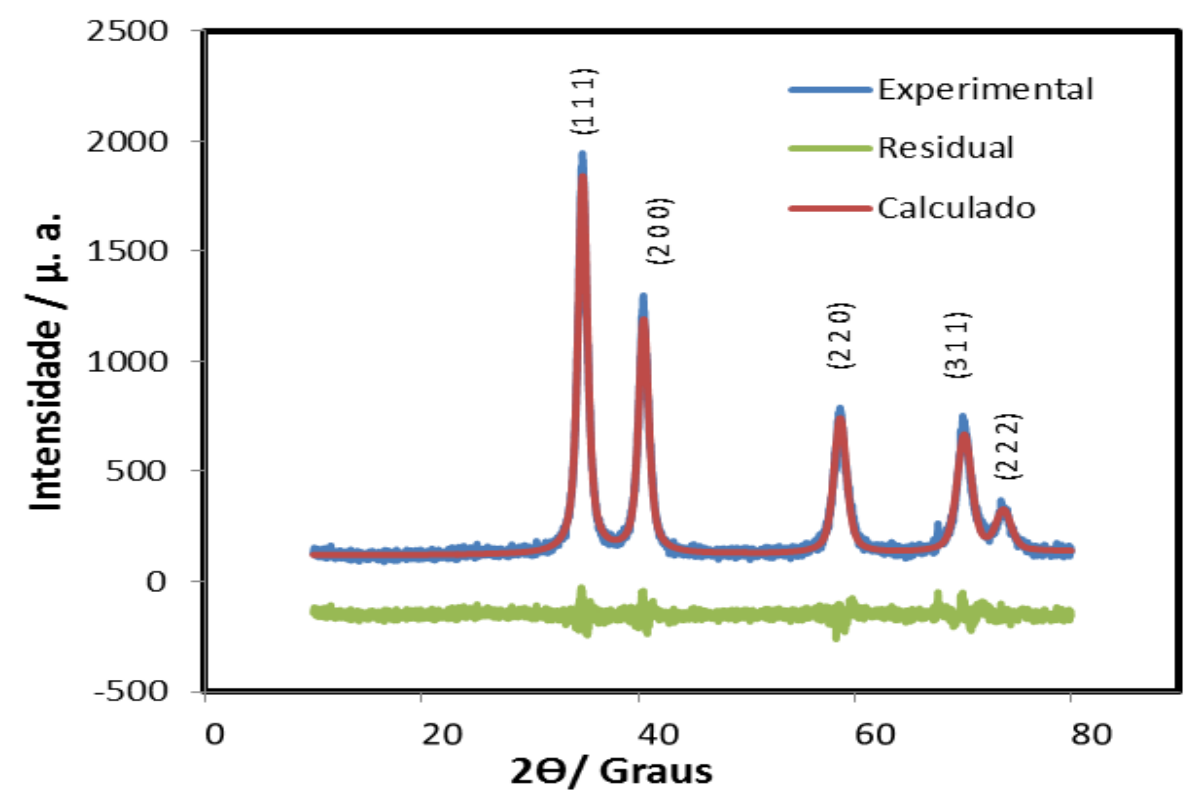

Figura 4. Espectro de Difração de Raios-X refinado pelo Método Rietveld.

Tabela 2. Dados do refinamento do carbeto de tântalo pelo Software Maud.

\begin{tabular}{|c|c|c|c|c|}
\hline Densidade & Volume de Célula & $\begin{array}{c}\text { Tamanho de } \\
\text { cristalito }\end{array}$ & Estrutura & Fase \\
\hline 14,5 & 4,4496 & 12,9 & CFC & 44497-ICSD \\
\hline
\end{tabular}

Foram realizadas medidas do tamanho de cristalito e microdeformação do pó de TaC. Os resultados obtidos dessas medidas são apresentados nas Tabelas 3 e 4. Como pode ser visto, o TaC sintetizado apresenta um tamanho de cristalito na ordem de nanômetros. Além disso, os valores de microdeformação são desprezíveis.

Tabela 3. Resultados do Tamanho médio de Cristalito obtido pelo Método de Scherrer

\begin{tabular}{|c|c|c|c|c|c|c|}
\hline Material & \multicolumn{6}{|c|}{ Posição (2Ө) } \\
\hline & 34,856 & 40,478 & 58,617 & 70,078 & 73,697 & $\begin{array}{c}\mathrm{Tm} \\
(\mathrm{nm})\end{array}$ \\
\hline $\mathrm{TaC}$ & 9,192934661 & 8,813669579 & 7,911484591 & 7,536157011 & 7,440182122 & 8,179 \\
\hline \multicolumn{7}{|c|}{ Obs: Tm = Tamanho médio de Cristalito } \\
\hline
\end{tabular}

Tabela 4. Resultados do Tamanho médio de Cristalito e Microdeformação obtido pelo Método de Williamson-Hall

\begin{tabular}{|c|c|c|}
\hline Material & $\mathrm{Tm}(\mathrm{nm})$ & $\mathrm{Md}(\%)$ \\
\hline $\mathrm{TaC}$ & 12,05 & 0,003250 \\
\hline \multicolumn{2}{|c|}{ Obs: $\mathrm{Tm}=$ Tamanho médio de Cristalito; $\mathrm{Md}=$ Microdeformação. } \\
\hline
\end{tabular}

\subsubsection{MEV do carbeto de tântalo}

Através das micrografias do carbeto de tântalo experimental obtidas pelo MEV, Figura $5 a$ e $5 b$, pode-se observar que, o produto resultante da carbonetação do precursor a partir de reação gás-sólido apresenta uma morfologia na sua maioria uniforme com partículas grandes formada de pequenas e finas partículas, algumas

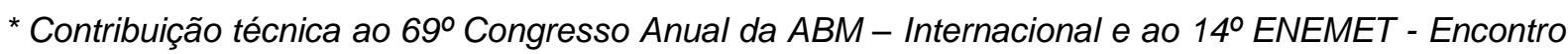
Nacional de Estudantes de Engenharia Metalúrgica, de Materiais e de Minas, 21 a 25 de julho de 2014, São Paulo, SP, Brasil.
} 


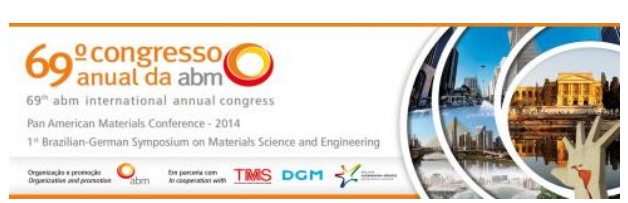

partículas e tamanho médio de cristalitos na ordem de nanômetros chegando a $12,05 \mathrm{~nm}$ e área superficial especifica de $19 \mathrm{~m} 2 / \mathrm{g}$. Estas características permitem 0 uso desse carbeto não apenas como reforço em metal duro e fabricação de ligas, mais também como catalisador. No entanto testes para trabalhos futuros devem ser realizados para comprovar sua possível aplicação em catálise.

\section{Agradecimentos}

Programa de Pós-Graduação em Ciências e Engenharia de Materiais- PPGCEM; Universidade Federal do Rio Grande do Norte- UFRN; Laboratório de Materiais Cerâmicos e Metais Especiais - LMCME; Laboratório de Materiais Nanoestruturados e Reatores Cataliticos - LAMNRC; Coordenação de Aperfeiçoamento de Pessoal de Nível Superior - CAPES.

\section{REFERÊNCIAS}

1 Vasconcelos BR, Morais AMV, Lopes FWB, Souza CP. Estudo da variação da concentração de $\mathrm{Cu}$ na/no $\mathrm{CuNb2O6}$ sintetizado a partir de reação sólido-sólido. In: Anais do XVIII Congresso Brasileiro de Engenharia Química - COBEQ; 2010; Paraná, Brasil. Paraná: COBEQ; 2010. 1 CD.

2 Medeiros FFP. Síntese de carbetos de tungstênio e nióbio a baixa temperatura, através de reação gás-sólido em reator de leito fixo [tese de doutorado em Engenharia Química]. Natal: Centro de Tecnologia, Departamento de Engenharia Química, Programa de Pós-Graduação em Engenharia Química, Universidade Federal do Rio Grande do Norte; 2002

3 Almeida RM, Pantano CG. Structural investigation of silica gel films by infrared spectroscopy. Journal of Applied Physics. 1990;68:4225-32.

4 Wada N, Kubo M, Maeda N, Akira M, Kojima K. Fluorescence property and dissolution site of Er3+ in Ta2O5 film prepared by sol-gel method and dip-coating technique. Journal of Materials Research. 2004;19(2):667-675.

5 Marta L, Zaharescu M, Macarovic C. Thermal and structural investigation of some oxalato-niobium complexes 3. strossium tris(oxalato)oxiniobate. Jornal of Thermal Analysis.1983;24:87-94.

6 Edelstein AS, Cammarata RC. Nanomaterials: synthesis, properties and aplications. London: Physics Publishing; 1996.

\footnotetext{
* Contribuição técnica ao 69ํ Congresso Anual da ABM - Internacional e ao 14ํㅡㄹ ENEMET - Encontro Nacional de Estudantes de Engenharia Metalúrgica, de Materiais e de Minas, 21 a 25 de julho de 2014, São Paulo, SP, Brasil.
} 